\title{
The Principle of Justifiable Granularity and an Optimization of Information Granularity Allocation as Fundamentals of Granular Computing
}

\author{
Witold Pedrycz*
}

\begin{abstract}
Granular Computing has emerged as a unified and coherent framework of designing, processing, and interpretation of information granules. Information granules are formalized within various frameworks such as sets (interval mathematics), fuzzy sets, rough sets, shadowed sets, probabilities (probability density functions), to name several the most visible approaches. In spite of the apparent diversity of the existing formalisms, there are some underlying commonalities articulated in terms of the fundamentals, algorithmic developments and ensuing application domains. In this study, we introduce two pivotal concepts: a principle of justifiable granularity and a method of an optimal information allocation where information granularity is regarded as an important design asset. We show that these two concepts are relevant to various formal setups of information granularity and offer constructs supporting the design of information granules and their processing. A suite of applied studies is focused on knowledge management in which case we identify several key categories of schemes present there.
\end{abstract}

Keywords-Information Granularity, Principle of Justifiable Granularity, Knowledge Management, Optimal Granularity Allocation

\section{INTRODUCTION}

Let us consider a system (process) for which constructed is a family of models. The system can be perceived from different points of view, observed over some time periods and analyzed at different levels of detail. Subsequently, the resulting models are built with various objectives in mind. They offer some particular, albeit useful views at the system. We are interested in forming a holistic model of the system by taking advantage of the individual sources of knowledge models, which have been constructed so far. When doing this, we are obviously aware that the sources of knowledge exhibit diversity and hence this diversity has to be taken into consideration and carefully quantified. No matter what the local models look like, it is legitimate to anticipate that the global model (say, the one formed at the higher level of hierarchy) is more general, abstract. Another point of interest is to engage the sources of knowledge in intensive and carefully orchestrated procedures of knowledge reconciliation and consensus building. Granularity of information [1-6, 21, 28, 29] becomes of paramount importance, both from the conceptual as well as algorithmic perspective, in the realization of granular fuzzy models. Subsequently, processing realized at the level of information granules gives rise to the discipline of Granular

\footnotetext{
Manuscript received May 20, 2011; accepted August 8, 2011.

Corresponding Author: Witold Pedrycz

* Department of Electrical \& Computer Engineering University of Alberta, Edmonton Canada and Systems Research Institute of the Polish Academy of Sciences Warsaw, Poland (pedrycz@ee.ualberta.ca)
} 
Computing [2]. We envision here a vast suite of formal approaches of fuzzy sets [15], rough sets [10-14], shadowed sets [16, 18, 19], probabilistic sets [9] and alike. Along with the conceptual setups, we also encounter a great deal of interesting and relevant ideas supporting processing of information granules. For instance, in the realm of rough sets we can refer to $[8,23,25,26,30]$ From the algorithmic perspective, fuzzy clustering [7], rough clustering, and clustering are regarded as fundamental development frameworks in which information granules are constructed.

The objective of this study is to introduce two conceptual and algorithmic underpinnings of Granular Computing, namely a principle of justifiable granularity and an optimal allocation of information granularity (Section 2 and 3). These two ideas are essential to the realization of granular fuzzy models. Four fundamental modes of knowledge management are identified (Section 4), which in turn are supplied with a selected category of problems of group decisionmaking (Section 5), granular compression applied to a reduction of rule-based architectures (Section 6), and knowledge transfer resulting in granular fuzzy models (Section 7).

\section{The Principle of Justifiable GranUlarity}

Here we are concerned with the formation of a single information granule $\Omega$ based on some experimental evidence being a set of a single-dimensional (scalar) numeric data, $\mathbf{D}=\left\{\mathrm{x}_{1}, \mathrm{x}_{2}, \ldots\right.$, $\left.\mathrm{x}_{\mathrm{N}}\right\}$.The principle of justifiable granularity [15] is concerned with a formation of a meaningful information granule based on available experimental evidence. Such construct has to adhere to the two intuitively compelling requirements:

(i) the numeric evidence accumulated within the bounds of $\Omega$ has to be as high as possible. By doing so, we anticipate that the existence of the information granule is well motivated (justified) as being reflective of the existing experimental data.

(ii) at the same time, the information granule should be as specific as possible meaning that it comes with a well-defined semantics. In other words, we would like to have $\Omega$ as detailed (specific) as possible.

While these two requirements are appealing, they have to be translated into some operational framework where the formation of the information granule can be realized. This framework depends upon the accepted formalism of information granulation, viz. a way in which information granules are described as sets, fuzzy sets, shadowed sets, rough sets, probabilistic granules and alike. To start with a simple and convincing constructs, let us treat $\Omega$ as an interval to be constructed. The first requirement is quantified by counting the number of data falling within the bounds of $\Omega$. In the simplest scenario, we can look at the cardinality of $\Omega$, namely card $\left\{\mathrm{x}_{\mathrm{k}} \in \Omega\right\}$. More generally, we can consider an increasing functional of the cardinality, say $\mathrm{f}_{1}\left(\operatorname{card}\left\{\mathrm{x}_{\mathrm{k}} \in \Omega\right\}\right)$. The simplest case concerns an identity functional, $\mathrm{f}_{1}(\mathrm{u})=\mathrm{u}$. The specificity of the information granule can be quantified by taking into account its size. The length of the interval $\Omega$ or a decreasing functional of the length, $f_{2}$, can serve as a sound measure of specificity. The lower the value of $f_{2}($ length $(\Omega))$, the higher the specificity is. It is apparent that the two requirements discussed above are in conflict. As illustrated in Figure 1, the increase in the evidence behind the information granule is associated with the decrease in its specificity.

Let us proceed with the detailed construct of interval information granules. We start with a 


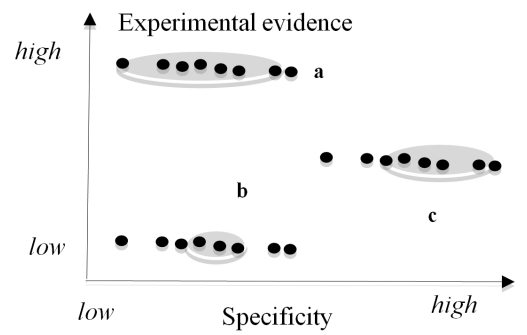

Fig. 1. Specificity versus experimental evidence of interval information granules: a conflicting nature of the requirements: a-high experimental evidence but a lack of specificity, b- low experimental evidence and high specificity, c- high specificity and acceptable experimental evidence

determination of the numeric representative of the set of data $\mathbf{D}$. A sound representative is its median, $\operatorname{med}(\mathbf{D})$, as it is a robust estimator of the sample and typically comes as one of the elements of $\mathbf{D}$. An information granule $\Omega$ is formed by forming its lower and upper bounds, denoted by a and b, respectively; refer also to Figure 2 .

The determination of the bounds is realized independently. In this sense, we can concentrate on the optimization of the upper bound (b). The calculations of the lower bound (a) are carried out in an analogous fashion. The length of $\Omega$, which quantifies the specificity of the information granule is given now as $|\operatorname{med}(\mathbf{D})-\mathrm{b}|$. More generally, we have $f_{2}(|\operatorname{med}(D)-b|)$ where $f_{2}$ is a nonincreasing (or decreasing) function. The cardinality of the information granule takes into account the elements of $\mathbf{D}$ positioned to the right from the median, card $\left\{\mathrm{x}_{\mathrm{k}} \in \Omega, \mathrm{x}_{\mathrm{k}}>\operatorname{med}(\mathbf{D})\right\}$. Again in general, we compute $\mathrm{f}_{1}\left(\operatorname{card}\left\{\mathrm{x}_{\mathrm{k}} \in \Omega, \mathrm{x}_{\mathrm{k}}>\operatorname{med}(\mathbf{D})\right\}\right)$. As the requirements of experimental evidence and specificity are in conflict, we can either resort ourselves to multiobjective optimization or consider a maximization of the product $\mathrm{V}=\mathrm{f}_{1} * \mathrm{f}_{2}$ whose optimization is to be realized with respect to the upper bound of the information granule, that is $V\left(b_{o p t}\right)=\max _{b>\operatorname{med}(\mathrm{D})} \mathrm{V}(\mathrm{b})$. In the same way, constructed is the lower bound of the information granule, $a_{\text {opt }} ; V\left(a_{\text {opt }}\right)=$ $\max _{a<\operatorname{med}(\mathrm{D})} \mathrm{V}(\mathrm{a})$. One among possible design alternatives, we can consider two functionals $\mathrm{f}_{1}$ and $\mathrm{f}_{2}$ assuming the following form

$$
\mathrm{f}_{1}(\mathrm{u})=\mathrm{u}
$$

and

$$
\mathrm{f}_{2}(\mathrm{u})=\exp (-\alpha \mathrm{u})
$$

where $\alpha$ is a positive parameter offering some flexibility in the produced information granule A. Its role is to calibrate an impact of the specificity criterion on the constructed information granule. Note that if $\alpha=0$ then $\mathrm{f}_{2}(\mathrm{u})=1$ and in this case the criterion of specificity is completely ruled out. In this case $b=x_{\max }$ with $x_{\max }$ being the largest element in $\mathbf{D}$. Higher values of $\alpha$ stress the increasing importance of the specificity criterion. Sufficiently high values of the parameter $\alpha$

$\Omega$

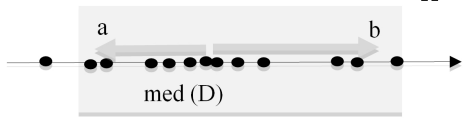

Fig. 2. Optimization of interval information granule $\Omega$ 
promote highly confined, numeric-like information granules.

As an illustrative example, let us consider a collection of data $\mathbf{D}$ governed by a Gaussian distribution with a standard deviation equal to $\sigma, \mathrm{p}(\mathrm{x}) \sim \mathrm{N}(0, \sigma)$. We are interested in the determination of the upper bound of $\Omega$. The maximized performance index is the product of $\int_{0}^{b} p(x) d x$ and the functional of the length $(b), \exp (-\alpha b), V(b)=\exp (-\alpha b) \int_{0}^{b} p(x) d x$. The plots of $V$ regarded as a function of "b" are included in Figure 3. We note that with the increase of the values of $\alpha$, the specificity of the resulting information granule gets higher (the interval becomes narrow); more specifically, $b=1.6$ and $b=1.2$ for the values of $\alpha$ equal to 0.1 and 0.3 , respectively.

Alluding to the format of the maximized multiplicative objective function $\mathrm{f}_{1} \mathrm{f}_{2}$, it is helpful to elaborate on the choice of the maximal value of $\alpha$. Note that for $\alpha=0.0$, we have $f_{2}$ equal identically to 0 and only the first component of $\mathrm{V}$ is used in the formation of the information granule; naturally the interval includes all experimental data. We observe the following two relationships; refer also to the notation displayed in Figure 4,

- if the upper bound a set to $\mathrm{x}_{1}$ returns $\operatorname{card}(\mathrm{A})=1$ and $\exp \left(-\alpha\left|\mathrm{x}_{1}-\mathrm{med}\right|\right)$.

- if the upper bound a is specified in such a way that embraces two the elements of the data closest to the median, $\left\{\mathrm{x}_{1}, \mathrm{x}_{2}\right\}$, we have $\operatorname{card}(\mathrm{A})=2$ and $\exp \left(-\alpha\left|\mathrm{x}_{2}-\mathrm{med}\right|\right)$.

Here the median is treated as a numeric representative of the experimental data. We also consider only the upper bound of the information granule; the construction in case of the lower bound is realized in the same manner as discussed here.

Let us request that the bound located at $\mathrm{x}_{1}$ maximizes the performance index. This means that the following inequality is satisfied:

$$
\exp \left(-\alpha\left|\mathrm{x}_{1}-\mathrm{med}\right|\right)>2 \exp \left(-\alpha\left|\mathrm{x}_{2}-\mathrm{med}\right|\right)
$$

Introducing the notation $\mathrm{d}_{1}=\mid \mathrm{x}_{1}$-med $\mid$ and $\mathrm{d}_{2}=\mid \mathrm{x}_{2}$-med $\mid$ we obtain

$$
\exp \left(-\alpha b_{1}\right)>2 \exp \left(-\alpha b_{2}\right)
$$

Its solution comes in the form $\exp \left(\alpha\left(d_{2}-d_{1}\right)\right)>2$. The maximal value of $\alpha, \alpha_{\max }$ is taken as

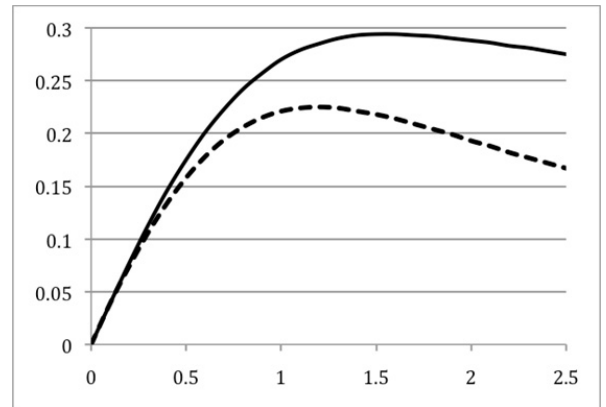

Fig. 3. Plots of $\mathrm{V}$ treated as a function of $\alpha$ and exhibiting some maxima for the data governed by the Gaussian distribution with $\sigma=1$ and $\alpha=0.1$ (solid line) and $\alpha=0.3$ (dotted line) 


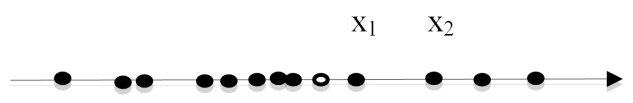

med

Fig. 4. A determination of a maximal value of $\alpha, \alpha_{\max }$; see details in the text

$\ln (2) /\left(d_{2}-d_{1}\right)$. It is associated with the maximal value of the membership grade that is made close to 1 , say $1-\varepsilon$ where e is a small positive value, say 0.01 . Practically, we can consider this value being equal to 0 . In this way we map the values of $\alpha$ used in the principle of justifiable granularity onto the $[0,1]$ interval of membership values

$$
\left[0, \alpha_{\max }\right] \rightarrow[0,1]
$$

In virtue of the monotonicity of the interval information granules with regard to the values of $\alpha$, each information granule can be associated with the corresponding value in $[0,1]$ (as expressed by (5)). Interestingly, a family of intervals indexed by the normalized values of $\alpha$ can be treated as a collection of $\alpha$-cuts. Subsequently, by varying the values of $\alpha$, we form the corresponding $\alpha$-cut and as a result, by making use of the representation theorem [15] arrive at an information granule in the form of a fuzzy set.

If the experimental data are governed by a certain probability density function $\mathrm{p}(\mathrm{x})$, the upper bound of the interval information granule is computed in a similar way as before. Here as there are no discrete numeric values $\mathrm{x}_{1}, \mathrm{x}_{2}, \ldots, \mathrm{x}_{\mathrm{N}}$, we consider a certain threshold level $\delta$ that links the upper bound of the information granule with a minimal acceptable level of experimental evidence. The corresponding relationships pertaining to the formulas presented above are expressed as follows

$$
\exp \left(-\alpha(\mathrm{m}+\delta-\mathrm{m}) \int_{\mathrm{m}}^{\mathrm{m}+\delta} \mathrm{p}(\mathrm{x}) \mathrm{dx}>\exp \left(-\alpha(\mathrm{m}+2 \delta-\mathrm{m}) \int_{\mathrm{m}}^{\mathrm{m}+2 \delta} \mathrm{p}(\mathrm{x}) \mathrm{dx}\right.\right.
$$

where " $\mathrm{m}$ " is the modal value of the corresponding probability density function $\mathrm{p}(\mathrm{x})$.

Finally, the expression for the maximal value of the parameter $\alpha$ comes in the form

$$
\alpha_{\max }=\ln \left(\int_{\mathrm{m}}^{\mathrm{m}+2 \delta} \mathrm{p}(\mathrm{x}) \mathrm{dx} / \int_{\mathrm{m}}^{\mathrm{m}+\delta} \mathrm{p}(\mathrm{x}) \mathrm{dx}\right) / 2 \delta
$$

The above construct can be augmented to situations where the individual data are associated with some weights (which could quantify their quality which may vary from one element to another). Given the data in the form $\left(\mathrm{x}_{\mathrm{i}}, \mathrm{w}_{\mathrm{i}}\right)$ where the weights $\mathrm{w}_{\mathrm{i}}$ assume values located in the $[0,1]$ interval, we reformulate the maximized performance index to be in the form

$$
\mathrm{V}(\mathrm{a})=\mathrm{f}_{1}\left(\sum_{\mathrm{k}=1}^{\mathrm{N}} \mathrm{w}_{\mathrm{i}}\right) \mathrm{f}_{2}(|\mathrm{a}-\mathrm{med}|)
$$

where " $m$ " is a weighted median whose computing uses the weighted data. 


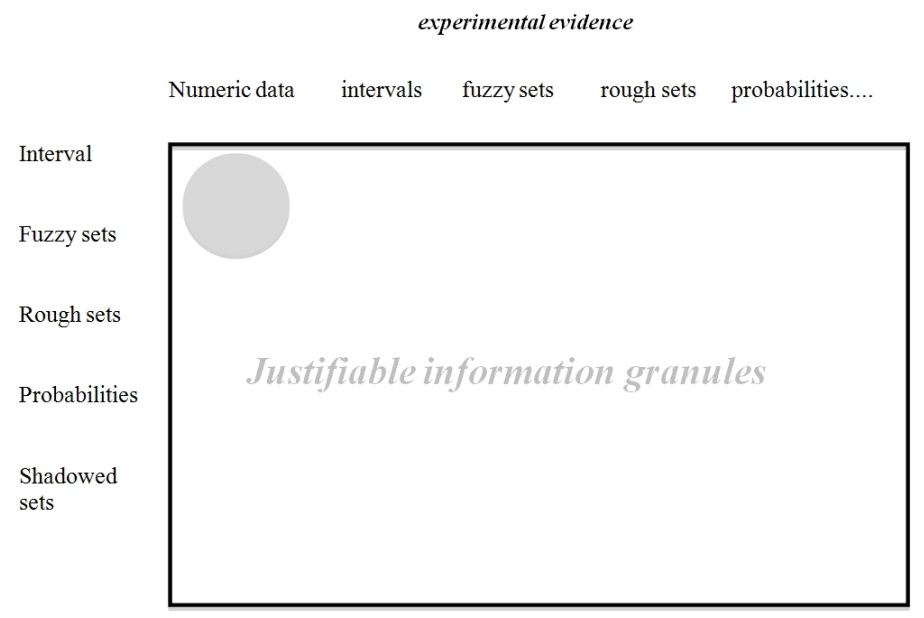

Fig. 5. The principle of justifiable granularity: a variety of environments of information granules and the nature of experimental evidence; highlighted are the alternatives for which detailed formulas have been derived

The principle of justifiable granularity covers a broad spectrum of scenarios- all of them can be arranged along two coordinates, Figure 5. The first one is concerned with the formal environment of information granules. The second one points at the nature of available experimental evidence (sets, fuzzy sets, etc.)

In our considerations we focused on the use of numeric evidence while constructed were interval like information granules and those expressed by fuzzy sets. The developed principle covers other cases presented in Fig. 5 with some modifications of the criteria pertinent to the specificity of the contemplated realization.

The realized information granule (either in a form of an interval or a fuzzy set) concerns onedimensional numeric data. The extension to the multidimensional case is straightforward by constructing a Cartesian product of the information granules formed for the individual variables. For instance, given an information granule A defined in $\mathbf{X}_{1}, \mathrm{~B}$ arising at $\mathbf{X}_{2}$ and $\mathrm{C}$ at $\mathbf{X}_{3}$, the result is in the form $\mathrm{A} \times \mathrm{B} \times \mathrm{C}$.

\section{Optimal Allocation of Information Granularity}

Information granularity is an important design asset. Information granularity allocated to the original numeric construct elevates a level of abstraction (generalizes) of the original construct developed at the numeric level. A way in which such an asset is going to be distributed throughout the construct or a collection of constructs to make the abstraction more efficient, is a subject to optimization.

Consider a certain mapping $\mathrm{y}=\mathrm{f}(\mathbf{x}, \mathbf{a})$ with a being a vector of parameters of the mapping. The mapping can be sought in a general way. One may think of a fuzzy model, neural network, polynomial, differential equation, linear regression, etc. The granulation mechanism $\boldsymbol{G}$ is applied to a giving rise to its granular counterpart, $\mathbf{A}=\boldsymbol{G}(\mathbf{a})$ and subsequently producing a granular mapping, $\mathrm{Y}=\boldsymbol{G}(\mathrm{f}(\mathbf{x}, \mathbf{a}))=\mathrm{f}(\mathbf{x}, \boldsymbol{G}(\mathbf{a}))=\mathrm{f}(\mathbf{x}, \mathbf{A})$. Given the diversity of the underlying constructs as well 


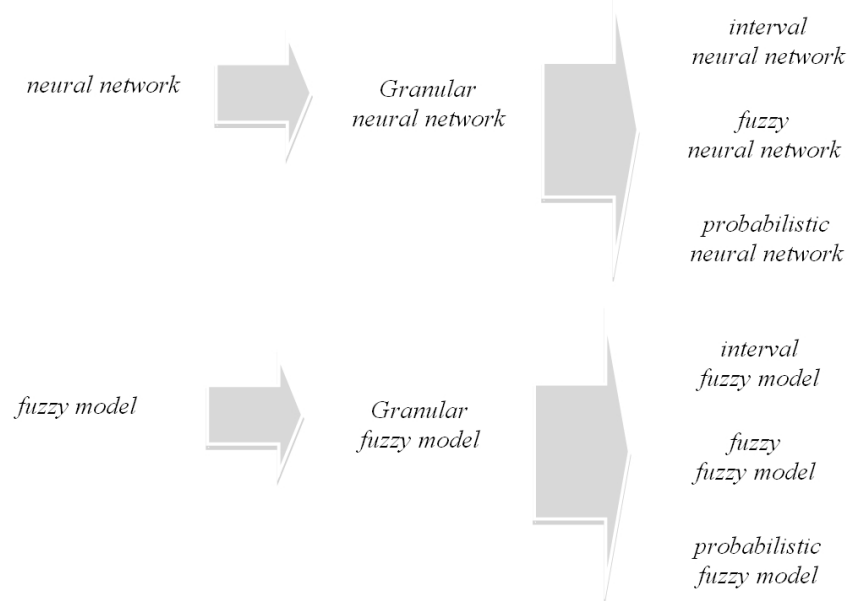

Fig. 6. A plethora of granular mappings; shown are examples of formalisms of information granularity applied to two selected categories of the mappings

as a variety of ways information granules can be formalized, we arrive at a suite of interesting constructs as illustrated in Figure 6. Note that through granulation, we form granular neural networks, say interval neural networks, fuzzy neural networks, probabilistic neural networks, etc.

There are a number of well-justified and convincing arguments behind elevating the level of abstraction of the existing constructs. Those include: an ability to realize various mechanisms of collaboration, quantification of variability of sources of knowledge considered, better modelling rapport with systems when dealing with nonstationary environments. In what follows, we will elaborate on the general categories of problems in which information granularity plays a pivotal role.

Information granularity provided to form a granular construct is an important design asset whose allocation throughout the mapping can be guided by certain optimization criteria. Let us discuss the underlying optimization problem in more detail. In addition to the mapping itself, we are provided with some experimental evidence in the form of input-output pairs $\left(\mathbf{x}_{\mathrm{k}}, \mathrm{t}_{\mathrm{k}}\right), \mathrm{k}=1$, $2, \ldots$, M. Given is a level of information granularity $\varepsilon, \varepsilon \in[0,1]$. We allocate the available level $\varepsilon$ to the parameters of the mapping, $\operatorname{dim}(\mathbf{a})=\mathrm{h}$, so that the some optimization criteria are satisfied while the allocation of granularity satisfies the following balance $\varepsilon=\sum_{\mathrm{i}=1}^{\mathrm{h}} \varepsilon_{\mathrm{i}}$ where non-negative $\varepsilon_{\mathrm{i}}$ is a level of information granularity associated with the i-th parameter of the mapping. All of the individual allocations are organized in a vector format $\left[\begin{array}{llll}\varepsilon_{1} & \varepsilon_{2} \ldots & \varepsilon_{\mathrm{h}}\end{array}\right]^{\mathrm{T}}$. The crux of the essence of the optimal allocation of granularity is displayed in Figure 7.

There are two optimization criteria to be considered in the optimization. The first one is concerned with the coverage of data $t_{k}$. For $\mathbf{x}_{k}$ we compute $Y_{k}=f\left(x_{k}, G(a)\right)$ and determine a degree of inclusion of $t_{k}$ in information granule $Y_{k}$, incl $\left(t_{k}, Y_{k}\right)=t_{k} \subset Y_{k}$. Then we compute an average sum of the degrees of inclusion taken over all data, that is $\frac{1}{M} \sum_{k=1}^{M}$ incl $\left(t_{k}, Y_{k}\right)$ Depending upon the formalism of information granulation, the inclusion returns a Boolean value in case of intervals (sets) or a certain degree of inclusion in case of fuzzy sets. 


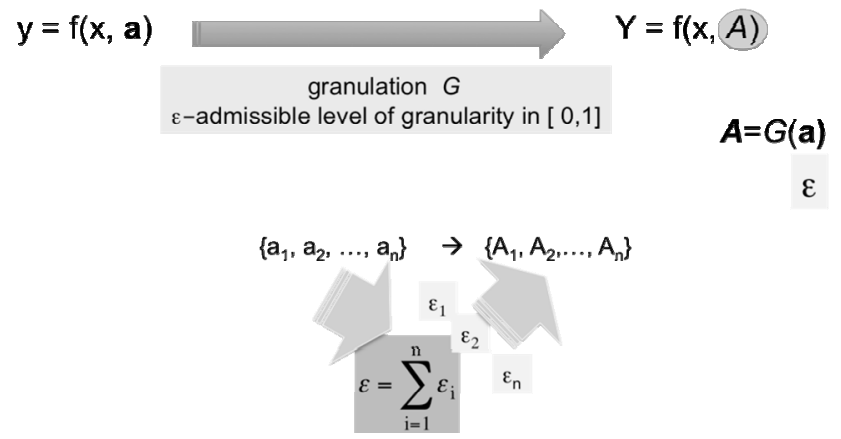

Fig. 7. From numeric to granular mapping formed through optimal allocation of granularity

The second criterion is focused on the specificity of $\mathrm{Y}_{\mathrm{k}}$ - we want it to be as high as possible. The specificity could be viewed as a decreasing function of the length of the interval in case of set -based information granulation. For instance, one can consider the inverse of the length of $Y_{k}$, say $1 /$ length $\left(Y_{k}\right)$, exp(-length $\left(Y_{k}\right)$ ), etc. In case of fuzzy sets, one consider the specificity involving the membership grades. The length of the fuzzy set $Y_{k}$ is computed by integrating the lengths of the $\beta$-cuts, $\int_{0}^{1} \operatorname{length}\left(\mathrm{Y}_{\mathrm{k}}^{\beta}\right) \beta \mathrm{d} \beta$

More formally, the two-objective optimization problem is formulated as follows.

Distribute (allocate) a given level of information granularity $\varepsilon$ so that the following two criteria are maximized

$$
\text { Maximize } \frac{1}{\mathrm{M}} \sum_{\mathrm{k}=1}^{\mathrm{M}} \operatorname{incl}\left(\mathrm{t}_{\mathrm{k}}, \mathrm{Y}_{\mathrm{k}}\right)
$$

Maximize $\mathrm{g}\left(\right.$ length $\left.\left(\mathrm{Y}_{\mathrm{k}}\right)\right)$ (where $\mathrm{g}$ is a decreasing function of its argument)

subject to $\varepsilon=\sum_{\mathrm{i}=1}^{\mathrm{h}} \varepsilon_{\mathrm{i}}$

A simpler, optimization scenario involves a single coverage criterion regarded as a single most essential criterion considered in the problem

$$
\text { Maximize } \quad \mathrm{Q}=\frac{1}{\mathrm{M}} \sum_{\mathrm{k}=1}^{\mathrm{M}} \operatorname{incl}\left(\mathrm{t}_{\mathrm{k}}, \mathrm{Y}_{\mathrm{k}}\right) \text { subject to } \varepsilon=\sum_{\mathrm{i}=1}^{\mathrm{h}} \varepsilon_{\mathrm{i}}
$$

There is an interesting monotonicity property: higher values of $\varepsilon$ lead to higher values of the maximized objective function see Figure 8.

By taking account the nature of the relationship shown in Figure 8, we can build some global view at the relationship that is independent from a specific value of $\varepsilon$ by taking an area under curve (AUC) computed as $\mathrm{AUC}=\int_{0}^{1} \mathrm{Q}(\varepsilon) \mathrm{d} \varepsilon$. The higher the value of the AUC, the higher the performance of the granular version of the mapping.

Information granularity can be realized in the setting of a certain information allocation protocol. Several main categories of such protocols can be envisioned: 


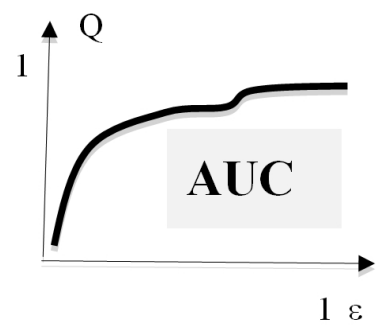

Fig. 8. Values of the coverage criterion $Q$ regarded as a function of the assumed level of granularity $\varepsilon$

$\mathrm{P}_{1}$ : uniform allocation of information granularity. This process is the simplest one and in essence does not call for any optimization mechanism. The numeric parameter $a$ of the mapping is replaced by the information granule $\boldsymbol{G}(a)$, which is the same in terms of the size and the distribution around $a$. If the formal setup of $\boldsymbol{G}$ concerns intervals then the numeric parameters of the mapping are replaced by intervals of the same length $(\varepsilon)$ and distributed symmetrically around the parameters of the mapping.

$\mathrm{P}_{2}$ : uniform allocation of information granularity with asymmetric position of intervals.

$\mathrm{P}_{3}$ : non-uniform allocation of information granularity with symmetrically distributed intervals of information granules.

$\mathrm{P}_{4}$ : non-uniform allocation of information granularity with asymmetrically distributed intervals of information granules.

$\mathrm{P}_{5}$ : An interesting point of reference, which is helpful in assessing a relative performance of the above methods, is to consider a random allocation of granularity. By doing this, one can quantify how the optimized and carefully thought out process of granularity allocation is superior over a purely random allocation process.

Depending upon the formalism of information granularity, the protocols can be made more specific. For instance, Figure 9 illustrates a collection of scenarios where information granules are represented as intervals.

The quality of the resulting granular mappings produced through invoking different granularity allocation protocols can be assessed by computing the resulting value of the AUC. In this way, we can establish a liner order within a collection of the protocols. In virtue of the increasing generality of the protocols, we can envision the following ordering $\mathrm{P}_{1} \prec \mathrm{P}_{2} \prec \mathrm{P}_{3} \prec \mathrm{P}_{4}$ where the notation $\mathrm{P}_{\mathrm{i}} \prec \mathrm{P}_{\mathrm{j}}$ denotes that $\mathrm{P}_{\mathrm{j}}$ is preferred over $\mathrm{P}_{\mathrm{i}}$ as producing higher values of the AUC.

We can think of fuzzy sets built around numeric values of the parameters where depending upon a certain the membership functions may exhibit symmetric or asymmetric character as well as come with various supports. In case of probabilistic information granules, one may talk about symmetric and asymmetric probability density functions with the modal values allocated to the numeric values of the parameters and standard deviations whose values vary from parameter to parameter. In total, we require a sum of the standard deviations to satisfy the predefined level of granularity that is $\sigma=\sum_{\mathrm{i}=1}^{\mathrm{h}} \sigma_{\mathrm{i}}$. 


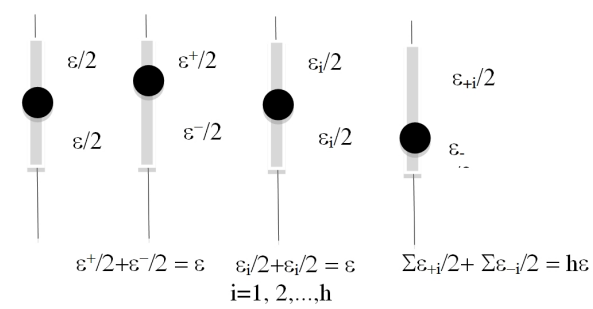

Fig. 9. Protocols $P_{1}-P_{4}$ of information granularity allocation - a case of interval information granulation

\section{Fundamental Modes of KnOWLedge Management}

Fuzzy models are sought as sources of knowledge. They interact in a variety of ways. It is advantageous to introduce a certain taxonomy, in which we delineate four modes of interaction as shown in Figure 10:

Aggregation of sources of knowledge The focal point concerns the formation of a global view about the system based upon the results (outcomes) produced by the individual models.

Granular compression A source of knowledge is compressed by forming a more compact model based on the original model. The compression brings about a concept of granularity- the compressed counterpart becomes more compact but inherently granular.

Building consensus The sources of knowledge (models) are actively engaged in the realization of a holistic view however in contrast to the previous category, there is an active involvement of the individual sources in the overall process in the sense they can constructively react to the global view created based on the individual and make some adjustments to themselves to increase a level of consensus. Some related studies are covered in [20,21].

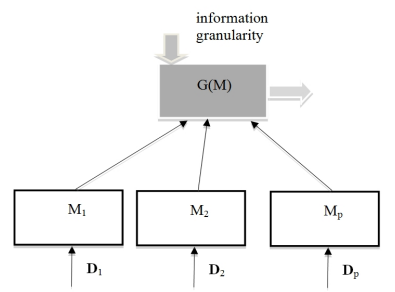

(a)

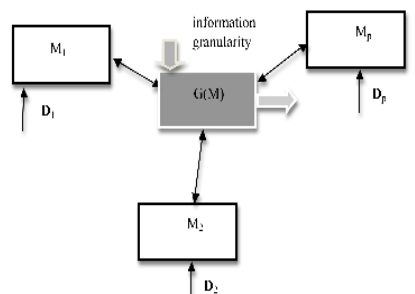

(b)

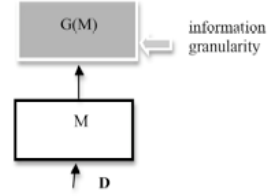

(c)

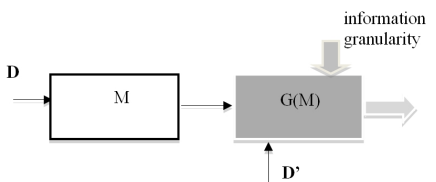

(d)

Fig. 10. Four modes of knowledge management: (a) aggregation, (b) consensus building, (c) granular compression, and (d) knowledge transfer 
Knowledge transfer A source of knowledge has been formed on some experimental evidence present in the past. New limited data originating from the similar process (which could evolve over time) stipulate that the existing source of knowledge could be used effectively by generalizing originally available model by making its granular. The granular model takes into account an effect of a partial relevance of the knowledge (model) acquired so far and through the allocation of information granules makes provisions with this regard.

In all these situations, the concept of information granules plays a pivotal role in several meaningful ways. First this concept facilitates or becomes a necessary prerequisite to the realization of knowledge management. Second, which is equally important, it quantifies the quality of the resulting constructs in the language of information granularity meaning that they become inherently made more abstract by incorporating information granules. Thus the specificity of the results is fully reflective of the diversity of the sources of knowledge and this helps assess the feasibility of interaction processes as well as undertake some necessary steps in cases the lack of specificity becomes too substantial (which speaks to the high level of diversity among sources knowledge being overly detrimental to the interaction there). Furthermore Figure 10 identifies a location of information granules as they emerge in the processes described above (the granular constructs are depicted by the shaded rectangular shapes).

In the first case, Fig.10 (a), granularity of information is reflective of the existing diversity of the sources of knowledge. In granular compression, the factor of granularity of information quantifies a trade-off between achieved level of compression and the associated level of abstraction of the resulting construct. In the two other cases, Fig.10 (c) - (d) information granularity is regarded as an important design asset which helps establish the underlying processes of consensus formation or quantifying knowledge transfer. The level of information granularity is introduced into the process externally and this design asset can be further optimized in terms of its allocation to the corresponding models involved in the scheme.

The granular models obtained as a result the interaction establish themselves at the higher level of abstraction in comparison with the available sources of knowledge. The term of granular fuzzy model is conceptually quite general as the realization depends upon a way in which information granules are expressed. Depending on this, we may talk about fuzzy fuzzy (fuzzy ${ }^{2}$ ) models, interval fuzzy models, rough fuzzy models, etc. There is a variety of ways in which the main categories of the above taxonomy manifest and give rise to numerous detailed realizations. In the following sections, we elaborate on some selected approaches being representative to the key categories of the taxonomy. Prior to that we elaborate on the idea of granularity allocation regarded as an essential optimization vehicle in the development of granular fuzzy models.

\section{Granular Analytic Hierarchy Process (AHP) Models}

This scenario is an example of granularity allocation where this assignment of granularity arises as a key component to facilitate collaboration. The Analytic Hierarchy Process (AHP) [24] is aimed at forming a vector of preferences for a finite set of $\mathrm{n}$ alternatives. These preferences are formed on a basis of a reciprocal matrix $R, R=\left[r_{i j}\right], i, j=1,2, \ldots, n$ whose entries are a result of pairwise comparisons of alternatives provided by a decision-maker. The quality of the result (reflecting the consistency of the judgment of the decision-maker) is expressed in terms of the following inconsistency index 


$$
v=\frac{\lambda_{\max }-n}{n-1}
$$

Where $\lambda_{\max }$ is the largest eigenvalue associated with the reciprocal matrix. The larger the value of this index is, the more significant level of inconsistency is associated with the preferences collected in the reciprocal matrix. Consider a group decision-making scenario where there is a group of decision makers resulting in a collection of reciprocal matrices each of them coming with a vector of preferences and the associated level of inconsistency.

Building consensus is about arriving at the preference vector that everybody is comfortable with. It is needless to say that to reach this state each player has to exercise a certain degree of flexibility and be ready to soften his position. Here information granularity comes into play. In a nutshell, we admit a granular (instead of numeric) realization of the reciprocal matrices where the granular entries of the matrices facilitate collaboration. Consider a group decision-making scenario in which there are c decision-makers and each of them comes with own preferences (preference vectors), $\mathbf{e}[1], \mathbf{e}[2], \ldots, \mathbf{e}[\mathbf{c}]$ obtained by running the AHP for the corresponding reciprocal matrix $R[1], R[2], \ldots, R[c]$. Furthermore the quality of preference vectors is quantified by the associated inconsistency index [i].

First, in the optimization problem, we bring all preferences close to each other and this goal is realized by adjusting the reciprocal matrices within the bounds offered by the admissible level of granularity provided to each decision-maker.

$$
\mathrm{Q}_{1}=\sum_{\mathrm{i}=1}^{\mathrm{c}}\left(1-v_{\mathrm{i}}\right)\|e[i]-\hat{\mathbf{e}}\|^{2}
$$

Where $\hat{\mathbf{e}}$ stands for the vector of preferences which minimizes the weighted sum of differences $\|$.$\| between \boldsymbol{e}[\mathrm{i}]$ and $\hat{\mathbf{e}}$. The detailed calculations depend on the form of the distance function used here. In particular, if we select the Euclidean distance, then the optimal vector of preferences $\hat{\mathbf{e}}$ is expressed as follows

$$
\hat{\mathbf{e}}=\frac{\sum_{\mathrm{i}=1}^{c}\left(1-v_{\mathrm{i}}\right) \mathbf{e}_{\mathrm{i}}}{\sum_{\mathrm{i}=1}^{\mathrm{c}}\left(1-v_{\mathrm{i}}\right)}
$$

Second, we increase the consistency of the reciprocal matrices and this improvement is realized at the level of individual decision-maker. The following performance index quantifies this aspect of collaboration

$$
\mathrm{Q}_{2}=\sum_{\mathrm{i}=1}^{\mathrm{c}} v_{\mathrm{i}}
$$

These are the two objectives to be minimized. If we consider the scalar version of the optimization problem, it can arise in the following additive format 


$$
\mathrm{Q}=\gamma \mathrm{Q}_{1}+\mathrm{Q}_{2}
$$

where $\gamma \geq 0$. The overall optimization problem reads now as follows

$$
\operatorname{Min}_{R[1], R[2], \ldots, R[\mathrm{c}]} \in \mathbf{G ( R )} Q
$$

where $\boldsymbol{G}(\mathrm{R})$ stands for the granular version of the of the reciprocal matrix. We require that the overall balance of the predefined level of granularity given in advance $\varepsilon$ is retained meaning that the following requirements

$$
\varepsilon=\sum_{\mathrm{i}=1}^{\mathrm{p}} \varepsilon_{\mathrm{i}}
$$

is satisfied. The summation is taken over all elements (p) of the reciprocal matrix, which assume values below 1 . The constraint applies to each reciprocal matrix so that for the given reciprocal matrix we arrive at elements $\varepsilon_{1}, \varepsilon_{2}, \ldots, \varepsilon_{c}$ that become a part of the overall optimization problem, meaning that (16) is expanded and reads now as

$$
\operatorname{Min}_{R[1], R[2], \ldots, R[\mathrm{c}]} \in \boldsymbol{P}(R) Q
$$

subject to the level of granularity constraint (17) articulated for each reciprocal matrix.

For the detailed discussion, the reader is referred to [17].

\section{GRANULAR COMPRESSION AND GRANULAR FUZZY RULE-BASED SYSTEMS}

The motivation behind the emergence of a granular generalization of fuzzy rules comes from a compactification (reduction) of an original set of rules. The underlying intuitively appealing idea is that to compensate for the reduction in the size of the rule base, we make the fuzzy sets standing in the remaining rules more abstract, viz. granular. We emphasize this development by using the term granular fuzzy rule. The reduced set of rules is composed of granular fuzzy sets, say fuzzy sets whose membership grades are described in terms of information granules, say intervals, fuzzy sets or probability density functions. Formally speaking, the original rule base $\left\{\mathrm{R}_{\mathrm{k}}\right\}=\left\{\right.$ if $\mathrm{A}_{\mathrm{k}}$ then $\left.\mathrm{B}_{\mathrm{k}}, \mathrm{k}=1,2, \ldots, \mathrm{N}\right\}$ composed of $\mathrm{N}$ rules is now reduced to the collection of "I" rules

$$
\text { -if } \boldsymbol{G}\left(\mathrm{A}_{\mathrm{i}}\right) \text { then } \mathrm{B}_{\mathrm{i}}
$$

$\mathrm{i} \in \mathbf{I}$. Here $\boldsymbol{G}\left(\mathrm{A}_{\mathrm{i}}\right)$ denotes the granular realization (generalization) of $\mathrm{A}_{\mathrm{i}}$. The nature of the formal framework of information granularity is not predefined in advance. The collection of indexes $\mathbf{I}=\left\{\mathrm{i}_{1}, \mathrm{i}_{2}, \ldots, \mathrm{i}_{\mathrm{I}}\right\}$ is specified as a subset if integers coming from $\mathbf{N}=\{1,2, \ldots, \mathbf{N}\}$. There is a separate optimization problem realized with this regard.

The quality of the granular rules can be expressed by counting how often the conclusion of the rule not being a part of the reduced set of rules is "covered" (contained) by the conclusion resulting from the reduced set of rules being now composed of granular fuzzy rules. More for- 
mally, let us consider $A=A_{j}$ where the rule "if $A_{j}$ then $B_{j}$ " is not a part of the index set $\mathbf{I}$. The fuzzy set $A$ is processed by the rules " if $\mathbf{G}\left(\mathrm{A}_{\mathrm{i}}\right)$ then $\mathrm{B}_{\mathrm{i}}$ " resulting in the interval-valued conclusion $\left[\mathrm{B}_{-}, \mathrm{B}_{+}\right]$. We count the elements of the conclusion space that is those, which satisfy the inclusion relationship $\mathrm{B}_{\mathrm{j}}(\mathrm{y}) \in\left[\mathrm{B}_{-}(\mathrm{y}), \mathrm{B}_{+}(\mathrm{y})\right]$. The process of repeated for all N-I rules that are outside the reduced rule set and a total count (s) is obtained. In an ideal situation, a ratio $\mathrm{s} /(\mathrm{N}$ I)*m) is equal to 1 , which becomes indicative of a complete inclusion of the conclusion of the original rule in the granular result of reasoning completed for the reduced rule base.

\section{Formation of GRANULAR FuZZy MOdels Through KNOWLEdge TRANSFER}

Let us consider a design scenario in which a fuzzy model, say a fuzzy neural network with logic neurons [15] has been developed based on some data D. Denote the model obtained in this way by $\mathrm{N}(\mathbf{x})$. Now we are supplied with another quite limited data, say $\mathbf{D}$ ' generated by the same or similar phenomenon, which entails that the characteristics of D' are not exactly the same as those describing $\mathbf{D}$ (for instance, the phenomenon could exhibit some non-stationary effect or there might be different conditions under which the data have been acquired, say increased level of noise or impact coming from auxiliary variables. The knowledge conveyed by the original model can be augmented and used instead of forming a new model based exclusively on the newly available data D'. The knowledge transfer (which in essence is represented by the model $\mathrm{N}$ ) manifests in the formation of the granular fuzzy model. The level of granularity present in the model formed in this manner is regarded to be an important design asset whose optimal allocation helps in an effective usage of knowledge already accommodated on basis of D. A convincing example of the realization of this concept comes with the design of granular fuzzy neural networks. The term granular stems from the fact that the original numeric values of the connections of the network are generalized to their granular counterparts. The connection $\mathrm{z}$ becomes replaced by the corresponding information granule, $\boldsymbol{G}(\mathrm{z})$. For instance, one forms information granules such as intervals that become distributed around the numeric values of the connections or some probability density functions, see Figure 8. The optimal allocation of granularity, viz. an allocation of the lengths of the intervals of the connections is realized in such a way that a certain criterion becomes optimized.

It is worth noting that knowledge transfer can be realized for any model; the allocation of granularity is concerned with its parameters.

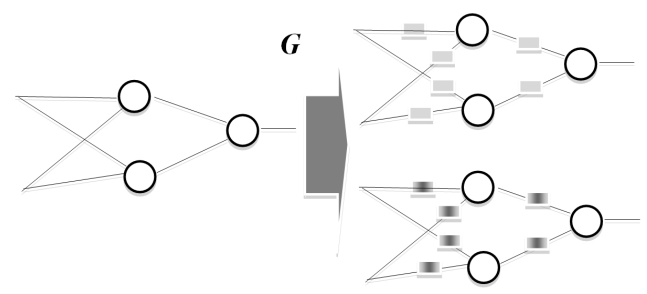

Fig. 11. From fuzzy neural network to its granular abstraction (generalization); small rectangular shapes point at the interval-valued character of the connections while shaded rectangular shapes depict the probability density functions of the granular connections 


\section{Conclusions AND Future DeVelopments}

Granular modelling calls for some fundamentals, a unified view at various formalisms contributing to the area, and ensuing principles addressing the ways of constructing and processing information granules in spite of a way they are formalized. This study brings forward the principle of justifiable granularity and the concept of optimal allocation of granularity in the design of intelligent systems. The first one is focused on the design of information granules. The second one highlights the role of information granules as a vehicle facilitating various facets of knowledge management especially those focused on collaborative schemes of knowledge reconciliation and consensus building.

We have highlighted several categories of representative design problems along with some general formulation of the ensuing optimization tasks without delving into the detailed algorithmic investigations. It is worth noting that the conceptual framework exhibits a significant level of generality and as such it may accommodate a variety of formal vehicles of information granulation as well as allow for a significant flexibility when it comes to specific developments. The idea of optimal allocation (distribution) of information granularity calls for more advanced techniques of optimization (that go far beyond gradient-based techniques). In particular, one can anticipate the usage of evolutionary of swarm optimization methods. In this sense, we start witnessing here yet another example of synergy of technologies of Computational Intelligence. Further comprehensive exploitation of methods of global optimization is of particular relevance in this setting.

\section{REFERENCES}

[1] B. Apolloni, S. Bassis, D. Malchiodi, W. Pedrycz, "Interpolating support information granules", Neurocomputing, 71, 13-15, 2008, 2433-2445.

[2] A.Bargiela, W. Pedrycz, Granular Computing: An Introduction, Kluwer Academic Publishers, Dordrecht, 2003.

[3] A. Bargiela, W. Pedrycz (eds.), Human-Centric Information Processing Through Granular Modelling, Springer -Verlag, Heidelberg, 2009.

[4] A. Bargiela, W. Pedrycz, "Granular mappings", IEEE Transactions on Systems, Man, and Cybernetics-part A, 35, 2, 2005, 292-297.

[5] A. Bargiela, W. Pedrycz, "A model of granular data: a design problem with the Tchebyschev FCM", Soft Computing, 9, 2005,155-163.

[6] A. Bargiela, W. Pedrycz, "Toward a theory of Granular Computing for human-centered information processing", IEEE Transactions on Fuzzy Systems, 16, 2, 2008, 320-330.

[7] J.C. Bezdek, Pattern Recognition with Fuzzy Objective Function Algorithms, Plenum Press, N. York, 1981.

[8] S. Calegari, D. Ciucci, "Granular computing applied to ontologies", Int. Journal of Approximate Reasoning, 51, 4, 2010, 391-409.

[9] K. Hirota, "Concepts of probabilistic sets", Fuzzy Sets and Systems, 5, 1, 1981, 31-46.

[10] K. Hirota, W. Pedrycz, "Characterization of fuzzy clustering algorithms in terms of entropy of probabilistic sets", Pattern Recognition Letters, 2, 4, 1984, 213-216.

[11] Z. Pawlak, Rough Sets: Theoretical Aspects of Reasoning about Data, System Theory, Kluwer Academic Publishers, Dordrecht, 1991.

[12] Z. Pawlak, "Rough sets and fuzzy sets", Fuzzy Sets and Systems, 17, 1, 1985, 99-102.

[13] Z. Pawlak, "A. Skowron, Rudiments of rough sets", Information Sciences, 177, 1, 1 2007, 3-27.

[14] Z. Pawlak, "A. Skowron, Rough sets: Some extensions", Information Sciences, 177, 1, 2007, 28-40.

[15] W. Pedrycz, F. Gomide, Fuzzy Systems Engineering: Toward Human-Centric Computing, John Wiley, Hoboken, NJ, 2007. 
[16] W. Pedrycz, "Shadowed sets: representing and processing fuzzy sets", IEEE Trans. on Systems, Man, and Cybernetics, Part B, 28, 1998, 103-109.

[17] W. Pedrycz, M. Song, "Analytic Hierarchy Process (AHP) in group decision making and its optimization with an allocation of information granularity", IEEE Trans.on Fuzzy Systems, 2011, to appear.

[18] W. Pedrycz, Shadowed sets: bridging fuzzy and rough sets, In: Rough Fuzzy Hybridization. A New Trend in Decision-Making, S.K. Pal, A. Skowron, (eds.), Springer Verlag, Singapore, 1999, 179-199.

[19] W. Pedrycz, Interpretation of clusters in the framework of shadowed sets, Pattern Recognition Letters, 26, 15, 2005, 2439-2449.

[20] W. Pedrycz, K. Hirota, “A consensus-driven clustering”, Pattern Recognition Letters, 29, 2008, 1333-1343.

[21] W. Pedrycz, P. Rai, "Collaborative clustering with the use of Fuzzy C-Means and its quantification", Fuzzy Sets and Systems, 159, 18, 2008, 2399-2427.

[22] W. Pedrycz, "The design of cognitive maps: A study in synergy of granular computing and evolutionary optimization”, Expert Systems with Applications, 37, 10, 2010, 7288-7294.

[23] Y. Qian, J. Liang, Y. Yao, C. Dang, "MGRS: A multi-granulation rough set”, Information Sciences, 180, 6, 2010, 949-970.

[24] T. L. Saaty, "How to handle dependence with the analytic hierarchy process", Mathematical Modelling, 9, 1987, 369-376.

[25] D. Ślęzak, "Degrees of conditional (in)dependence: A framework for approximate Bayesian networks and examples related to the rough set-based feature selection", Information Sciences, 179, 3, 2009, 197-209.

[26] R. W. Swiniarski, A. Skowron, "Rough set methods in feature selection and recognition", Pattern Recognition Letters, 24, 6, 2003, 833-849.

[27] W-Z. Wu, Y. Leung, Theory and applications of granular labeled partitions in multi-scale decision tables, Information Sciences, In Press, Available online 10 May, 2011.

[28] L.A. Zadeh, "Towards a theory of fuzzy information granulation and its centrality in human reasoning and fuzzy logic", Fuzzy Sets and Systems, 90, 1997, 111-117.

[29] L.A. Zadeh, "From computing with numbers to computing with words-from manipulation of measurements to manipulation of perceptions", IEEE Trans. on Circuits and Systems, 45, 1999, 105-119.

[30] X. Zhang, Y. Yao, H. Yu, "Rough implication operator based on strong topological rough algebras", Information Sciences, 180, 19, 2010, 3764-3780.

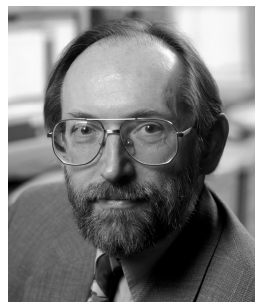

\section{Witold Pedrycz}

$\mathrm{He}$ is Professor and Canada Research Chair (CRC - Computational Intelligence) in the Department of Electrical and Computer Engineering, University of Alberta, Edmonton, Canada. He is also with the Systems Research Institute of the Polish Academy of Sciences, Warsaw, Poland. In 2009 Dr. Pedrycz was elected a foreign member of the Polish Academy of Sciences. He main research directions involve Computational Intelligence, fuzzy modeling and Granular Computing, knowledge discovery and data mining, fuzzy control, pattern recognition, knowledge-based neural networks, relational computing, and Software Engineering. He has published numerous papers in this area. He is also an author of 15 research monographs covering various aspects of Computational Intelligence and Software Engineering. Witold Pedrycz has been a member of numerous program committees of IEEE conferences in the area of fuzzy sets and neurocomputing.

Dr. Pedrycz is intensively involved in editorial activities. He is an Editor-in-Chief of Information Sciences and Editor-in-Chief of IEEE Transactions on Systems, Man, and Cybernetics - part A. He currently serves as an Associate Editor of IEEE Transactions on Fuzzy Systems and a number of other international journals. In 2007 he received a prestigious Norbert Wiener award from the IEEE Systems, Man, and Cybernetics Council. He is a recipient of the IEEE Canada Computer Engineering Medal. In 2009 he has received a Cajastur Prize for Soft Computing from the European Centre for Soft Computing for "pioneering and multifaceted contributions to Granular Computing". 